\title{
Sequentially perfect and uniform one-factorizations of the complete graph
}

\author{
Jeffrey H. Dinitz \\ Mathematics and Statistics \\ University of Vermont, Burlington, VT, USA 05405 \\ Jeff.Dinitz@uvm.edu \\ Peter Dukes \\ Mathematics and Statistics \\ University of Victoria, Victoria, BC, Canada V8W 3P4 \\ dukes@oddjob.math.uvic.ca \\ Douglas R. Stinson \\ School of Computer Science \\ University of Waterloo, Waterloo, ON, Canada N2L 3G1 \\ dstinson@uwaterloo.ca
}

Submitted: Aug 23, 2004; Accepted: Oct 12, 2004; Published: Jan 7, 2005

Mathematics Subject Classifications: 05C70

\begin{abstract}
In this paper, we consider a weakening of the definitions of uniform and perfect one-factorizations of the complete graph. Basically, we want to order the $2 n-1$ one-factors of a one-factorization of the complete graph $K_{2 n}$ in such a way that the union of any two (cyclically) consecutive one-factors is always isomorphic to the same two-regular graph. This property is termed sequentially uniform; if this two-regular graph is a Hamiltonian cycle, then the property is termed sequentially perfect. We will discuss several methods for constructing sequentially uniform and sequentially perfect one-factorizations. In particular, we prove for any integer $n \geq 1$ that there is a sequentially perfect one-factorization of $K_{2 n}$. As well, for any odd integer $m \geq 1$, we prove that there is a sequentially uniform one-factorization of $K_{2^{t} m}$ of type $(4,4, \ldots, 4)$ for all integers $t \geq 2+\left\lceil\log _{2} m\right\rceil$ (where type $(4,4, \ldots, 4$ ) denotes a two-regular graph consisting of disjoint cycles of length four).
\end{abstract}

\section{Introduction}

A one-factor of a graph $G$ is a subset of its edges which partitions the vertex set. A onefactorization of a graph $G$ is a partition of its edges into one-factors. Any one-factorization 
of the complete graph $K_{2 n}$ has $2 n-1$ one-factors, each of which has $n$ edges. For a survey of one-factorizations of the complete graph, the reader is referred to [10], [14] or [15].

A one-factorization $\left\{F_{0}, \ldots, F_{2 n-2}\right\}$ of $K_{2 n}$ is sequentially uniform if the one-factors can be ordered $\left(F_{0}, \ldots, F_{2 n-2}\right)$ so that the graphs with edge sets $F_{i} \cup F_{i+1}$ (subscripts taken modulo $2 n-1$ ) are isomorphic for all $0 \leq i \leq 2 n-2$. Since the union of two one-factors is a 2-regular graph which is 2-edge-colorable, it is isomorphic to a disjoint union of even cycles. We say the multiset $T=\left(k_{1}, \ldots, k_{r}\right)$ is the type of a sequentially uniform one-factorization if $F_{i} \cup F_{i+1}$ is isomorphic to the disjoint union of cycles of lengths $k_{1}, \ldots, k_{r}$, where $k_{1}+\cdots+k_{r}=2 n$. When the union of every two consecutive one-factors is a Hamiltonian cycle, the one-factorization is said to be sequentially perfect.

The idea to consider orderings of the one-factors in a one-factorization of $K_{2 n}$ is not entirely academic. In fact, an ordered one-factorization of $K_{2 n}$ is a schedule of play for a round-robin tournament (played in $2 n-1$ rounds). Round-robin tournaments possessing certain desired properties have been studied (see [15, Chapter 5], or [7]); however, to our knowledge, round robin tournaments with this "uniform" property have not been considered previously.

The definition above is a relaxation of the definition of uniform (perfect) one-factorization of $K_{2 n}$, which requires that the union of any two one-factors be isomorphic (Hamiltonian, respectively). Much work has been done on perfect one-factorizations of $K_{2 n}$; for a survey, see Seah [13]. Perfect one-factorizations of $K_{2 n}$ are known to exist whenever $n$ or $2 n-1$ is prime, and when $2 n=16,28,36,40,50,126,170,244,344,730,1332,1370$, 1850, 2198, 3126, 6860, 12168, 16808, and 29792 (see [1]). Recently a few new perfect one-factorizations have been found, the smallest of which is in $K_{530}$ (see $[4,9,16]$ ); however before this, no new perfect one-factorization of $K_{2 n}$ had been found since $1992([17])$. The smallest value of $2 n$ for which the existence of a perfect one-factorization of $K_{2 n}$ is unknown is $2 n=52$. We will show that sequentially perfect one-factorizations are much easier to produce and indeed we will produce a sequentially perfect one-factorization of $K_{2 n}$ for all $n \geq 1$.

Various uniform one-factorizations have been constructed from Steiner triple systems, [10]. For instance, when $n=2^{m}$ for some positive $m$, the so-called binary projective Steiner triple systems provide a construction of uniform one-factorizations of $K_{2 n}$ of type $(4,4, \ldots, 4)$. There are also sporadic examples of perfect Steiner triple systems, [8], which give rise to uniform one-factorizations of type $(2 n-4,4)$. When $v=3^{m}$, uniform onefactorizations $(4,6, \ldots, 6)$ exist (these are Steiner one-factorizations from Hall triple systems) and when $p$ is an odd prime there is a uniform one-factorization of $K_{p^{s}+1}$ of type $(p+1,2 p, \ldots, 2 p)$ which arises from the elementary abelian $p$-group (see [10]).

The remainder of this paper is organized as follows. In Section 2, we review the classical "starter" construction for one-factorizations and we show that sequentially perfect onefactorizations of $K_{2 n}$ exist for all $n$. In Section 3, we summarize existence results obtained by computer for small orders. In Section 4 , we investigate the construction of sequentially uniform one-factorizations from so-called quotient starters in noncyclic abelian groups. Here we obtain interesting number-theoretic conditions that determine if the resulting one-factorizations can be ordered so that they are sequentially uniform. In Section 5, 
we present a recursive product construction which yields infinite classes of sequentially uniform one-factorizations of $K_{2^{t} m}$ of type $(4,4, \ldots, 4)$, for any odd integer $m$.

\section{Starters}

We describe our main tool for finding sequentially uniform one-factorizations. Let $\Gamma$ be an abelian group of order $2 n-1$, written additively. A starter in $\Gamma$ is a set of $n-1$ pairs $S=\left\{\left\{x_{1}, y_{1}\right\}, \ldots,\left\{x_{n-1}, y_{n-1}\right\}\right\}$ such that every nonzero element of $\Gamma$ appears as some $x_{i}$ or $y_{i}$, and also as some difference $x_{j}-y_{j}$ or $y_{j}-x_{j}$. Let $S^{*}=S \cup\{\{0, \infty\}\}$ and define $x+\infty=\infty+x=\infty$ for all $x \in \Gamma$. Then $\left\{S^{*}+x: x \in \Gamma\right\}$ forms a one-factorization of $K_{2 n}$ (with vertex set $\Gamma \cup\{\infty\}$ ).

Many of the known constructions for (uniform and perfect) one-factorizations use starters in this way. In our first lemma we note the connection between starter-induced one-factorizations and sequentially uniform one-factorizations. Clearly, the order in which the 1-factors are listed is essential to the type of a sequentially uniform one-factorization. Thus we will sometimes refer to ordered one-factorizations in this context. Whenever we discuss sequentially uniform one-factorizations, we will always give the 1-factor ordering.

Lemma 2.1. Let $S$ be a starter in $\mathbb{Z}_{2 n-1}$ with $n \geq 1$. Then the ordered one-factorization of $K_{2 n}$ generated by $S$, namely $\left(S^{*}, S^{*}+1, S^{*}+2, \ldots, S^{*}+(2 n-2)\right)$ is sequentially uniform.

Proof: For any $x \in \mathbb{Z}_{2 n-1}$, we have $\left(S^{*}+x\right) \cup\left(S^{*}+(x+1)\right)=x+\left(S^{*} \cup\left(S^{*}+1\right)\right)$, so all unions of two consecutive one-factors in the given order are isomorphic.

Remark: When $\operatorname{gcd}(k, 2 n-1)=1$, the ordering $\left(S^{*}, S^{*}+k, S^{*}+2 k, \ldots, S^{*}+(2 n-2) k\right)$ of the same one-factorization is also sequentially uniform. Note, however, that it is not necessarily of the same type as the ordered one-factorization $\left(S^{*}, S^{*}+1, S^{*}+2, \ldots, S^{*}+\right.$ $(2 n-2))$.

The most well-known one-factorization of $K_{2 n}$ (called $G K(2 n)$ ) is generated from the patterned starter $P=\left\{\{x,-x\}: x \in \mathbb{Z}_{2 n-1}\right\}$ in the cyclic group $\mathbb{Z}_{2 n-1}$. It is known when $2 n-1$ is prime that $G K(2 n)$ is a perfect one-factorization and, in general, $G K(2 n)$ is a uniform one-factorization for all $n \geq 1$. The cycle lengths in $P^{*} \cup\left(P^{*}+k\right)$ for $k \in \mathbb{Z}_{2 n-1} \backslash\{0\}$ are now given.

Lemma 2.2. Let $P$ be the patterned starter in $\mathbb{Z}_{2 n-1}$ with $n \geq 1$. Let $k \in \mathbb{Z}_{2 n-1} \backslash\{0\}$ with $\operatorname{gcd}(2 n-1, k)=d$. Then $P^{*} \cup\left(P^{*}+k\right)$ consists of a cycle of length $1+(2 n-1) / d$ and $(d-1) / 2$ cycles of length $2(2 n-1) / d$.

Proof: The cycle through infinity is $(\infty, 0,2 k,-2 k, 4 k,-4 k, \ldots,-k, k)$, which has length $1+(2 n-1) / d$. All other cycles (if any) are of the form

$$
(i,-i, 2 k+i,-2 k-i, 4 k+i,-4 k-i, \ldots,-2 k+i, 2 k-i),
$$

for $1 \leq i<d$.

Combining Lemmas 2.1 and 2.2 (with $d=1$ ) we have the following result. 
Theorem 2.3. For every $n \geq 1$ there exists a sequentially perfect one-factorization of $K_{2 n}$.

Contrast this with the known results for perfect one-factorizations: the sporadic small values mentioned in the Introduction, and only two infinite classes (each of density zero).

\section{Small orders}

The one-factorizations of $K_{4}$ and $K_{6}$ are unique and in each case they are perfect. Hence both are sequentially perfect (the only possible type in these small cases). The onefactorization of $K_{8}$ obtained from the unique Steiner triple system of order 7 has type $(4,4)$ while $G K(8)$ is a perfect one-factorization. Hence there exist sequentially uniform one-factorizations of $K_{8}$ of all possible types.

We have checked all starters in $\mathbb{Z}_{9}$ by computer and report that no ordering of the translates of any of these starters yields a sequentially uniform one-factorization of $K_{10}$ of type $(4,6)$. However, there does exist a uniform one-factorization of type $(4,6)$ (it is one-factorization \#1 in the list of all 396 non-isomorphic one-factorizations of $K_{10}$ given in $[1$, p. 655$])$. Clearly this is also sequentially uniform of type $(4,6)$ under any ordering of the one-factors. From Theorem 2.3 there exists a sequentially perfect one-factorization of $K_{10}$. Thus sequentially uniform one-factorizations of $K_{10}$ exist for both possible types.

Obviously, the ordering of the one-factors can affect the type of the 2-factors formed from consecutive 1-factors in an ordered one-factorization. Given a starter $S$ in $\mathbb{Z}_{2 n-1}$, let $F_{S}(k)$ denote the ordered one-factorization $\left(S^{*}, S^{*}+k, S^{*}+2 k, \ldots, S^{*}+(2 n-2) k\right)$ of $K_{2 n}$. In the following examples we discuss sequentially uniform one-factorizations in $K_{12}$ and $K_{14}$. In $\mathbb{Z}_{13}$ we will give one starter which induces all possible types of ordered one-factorizations when different orderings are imposed on translates of that starter.

Example 3.1. Given the following starter in $\mathbb{Z}_{11}$,

$$
S=\{\{1,2\},\{3,8\},\{4,6\},\{5,9\},\{7,10\}\},
$$

$F_{S}(1)$ is sequentially uniform of type $(6,6), F_{S}(2)$ is sequentially uniform of type $(4,8)$ and $F_{S}(3)$ is sequentially uniform of type (12).

By checking all starters in $\mathbb{Z}_{11}$, we found that no ordering of any of the one-factorizations formed by these starters gave a sequentially uniform one-factorization of type $(4,4,4)$. However, Figure 1 provides a non-starter-induced ordered one-factorization which is sequentially uniform of this type.

In [12] it is found that there exist exactly five nonisomorphic perfect one-factorizations of $K_{12}$ and in [2] a uniform one-factorization of type $(6,6)$ is given. From the enumeration in [5], it is known that there exist no other uniform one-factorizations of $K_{12}$. Hence it is noteworthy that $F_{S}(2)$ (defined in Example 3.1) gives a sequentially uniform one-factorization of $K_{12}$ of type $(8,4)$ and Figure 1 gives a sequentially uniform one-factorization of type $(4,4,4)$. 
Figure 1: A sequentially uniform one-factorization of $K_{12}$ with type $(4,4,4)$

$$
\begin{aligned}
& F_{0}:\{\{0,1\},\{2,6\},\{3,4\},\{7,9\},\{8,10\},\{5,11\}\} \\
& F_{1}:\{\{0,2\},\{1,6\},\{3,9\},\{4,7\},\{5,10\},\{8,11\}\} \\
& F_{2}:\{\{0,3\},\{1,4\},\{5,8\},\{6,7\},\{2,9\},\{10,11\}\} \\
& F_{3}:\{\{0,4\},\{1,3\},\{2,8\},\{7,10\},\{6,11\},\{5,9\}\} \\
& F_{4}:\{\{0,5\},\{1,2\},\{3,8\},\{4,9\},\{6,10\},\{7,11\}\} \\
& F_{5}:\{\{0,8\},\{1,7\},\{2,11\},\{3,5\},\{4,6\},\{9,10\}\} \\
& F_{6}:\{\{0,6\},\{1,5\},\{2,10\},\{4,8\},\{3,7\},\{9,11\}\} \\
& F_{7}:\{\{0,7\},\{1,10\},\{2,5\},\{3,6\},\{4,11\},\{8,9\}\} \\
& F_{8}:\{\{0,9\},\{1,11\},\{2,3\},\{4,10\},\{5,6\},\{7,8\}\} \\
& F_{9}:\{\{0,11\},\{1,9\},\{2,4\},\{3,10\},\{6,8\},\{5,7\}\} \\
& F_{10}:\{\{0,10\},\{1,8\},\{2,7\},\{3,11\},\{4,5\},\{6,9\}\}
\end{aligned}
$$

Example 3.2. The following starter in $\mathbb{Z}_{13}$,

$$
S=\{\{1,10\},\{2,3\},\{4,9\},\{5,7\},\{6,12\},\{8,11\}\}
$$

yields sequentially uniform one-factorizations of $K_{14}$ of all possible types: namely (14), $(10,4),(8,6)$, and $(6,4,4)$. Specifically, $F_{S}(3)$ is sequentially uniform of type $(6,4,4)$, $F_{S}(1)$ is sequentially uniform of type $(8,6), F_{S}(2)$ is sequentially uniform of type $(10,4)$ and $F_{S}(5)$ is sequentially uniform of type (14).

For large $n$, there are many more possible types than there are translates, so the starter in Example 3.2 is of particular interest. In the Appendix we give examples of sequentially uniform one-factorizations of $K_{2 n}$ of all possible types, for $14 \leq 2 n \leq 24$.

\section{Starters in non-cyclic groups}

Many uniform and perfect one-factorizations are known to be starter-induced over a noncyclic group; for example, see [6]. So it is natural to also expect sequentially uniform one-factorizations where the ordering is not cyclic. In this section we give a numerical condition that determines when certain starter-induced one-factorizations over non-cyclic groups are sequentially uniform.

Let $q$ be an odd prime-power (not a prime) and write $q=2 r t+1$, where $t$ is odd. In order to eliminate trivial cases, we will assume that $t>1$. Suppose $\omega$ is a generator of the multiplicative group of $\mathbb{F}_{q}$ and let $Q$ be the subgroup (of order $t$ ) generated by $\omega^{2 r}$. Suppose the cosets of $Q$ are $C_{i}=\omega^{i} Q, i=0, \ldots, 2 r-1$. A starter $S$ in $\mathbb{F}_{q}$ is said to be an $r$-quotient starter if, whenever $\{x, y\},\left\{x^{\prime}, y^{\prime}\right\} \in S$ with $x, x^{\prime} \in C_{i}$, it holds that $y / x=y^{\prime} / x^{\prime}$. An $r$-quotient starter $S$ can be completely described by a list of quotients $\left(a_{0}, \ldots, a_{r-1}\right)$, such that

$$
S=\left\{\left\{x, a_{i} x\right\}:\left(a_{i}-1\right) x \in C_{i}, i=0, \ldots, r-1\right\} .
$$


It is not hard to see that $S^{*} \cup\left(S^{*}+x\right)$ is isomorphic to $S^{*} \cup\left(S^{*}+y\right)$ whenever $x / y \in C_{0} \cup C_{r}$. It follows that every 1-quotient starter yields a uniform one-factorization. We now show that, although $r$-quotient starters might not generate uniform one-factorizations when $r>1,[6]$, the resulting one-factorizations usually can be ordered in such a way that they are sequentially uniform.

Theorem 4.1. Suppose $q=p^{d}$ is an odd prime-power (with p prime and $d>1$ ) such that $q=2 r t+1$ and $t>1$ is odd. Let $S$ be any $r$-quotient starter in $\mathbb{F}_{q}$. Then the one-factorization generated by $S$ can be ordered to be sequentially uniform if and only if the multiplicative order of $p$ modulo $t$ is equal to $d$.

Proof: Let $q=p^{d}=2 r t+1$ with $t$ odd. $C_{0}$ is the multiplicative subgroup of $\mathbb{F}_{q}^{*}$ generated by a primitive th root of 1 in $\mathbb{F}_{q}$, say $\alpha$. The splitting field of $x^{t}-1$ over $\mathbb{F}_{p}$ is $\mathbb{F}_{p^{e}}$, where $e$ is the smallest positive integer such that $p^{e} \equiv 1(\bmod t)$. Hence the extension field $\mathbb{F}_{p}(\alpha)=\mathbb{F}_{q}$ if and only if the multiplicative order of $p$ modulo $t$, which we denote by $\operatorname{ord}_{t}(p)$, is equal to $d$.

Suppose that $\operatorname{ord}_{t}(p)=d$. Then $\mathbb{F}_{p}(\alpha)=\mathbb{F}_{q}$ and $1, \alpha, \ldots, \alpha^{d-1}$ is a basis of $\mathbb{F}_{q}$ over $\mathbb{F}_{p}$. Therefore, every element $x \in \mathbb{F}_{q}$ can be expressed uniquely as a $d$-tuple $\left(x_{1}, \ldots, x_{d}\right) \in$ $\left(\mathbb{Z}_{p}\right)^{d}$, where

$$
x=\sum_{i=1}^{n} x_{i} \alpha^{i-1} .
$$

Now, consider the graph on vertex set $\left(\mathbb{Z}_{p}\right)^{d}$ in which two vertices are adjacent if and only if they agree in $d-1$ coordinates and their values in the remaining coordinate differ by 1 modulo $p$ (this is a Cayley graph of the elementary abelian group of order $p^{d}$ ). It is not hard to check that this graph has a hamiltonian cycle, say $C=\left(y_{1}, y_{2}, \ldots, y_{p^{d}}, y_{1}\right)$. The cycle $C$ provides the desired ordering of $\mathbb{F}_{q}$ because the difference between any two consecutive elements $y_{i}$ and $y_{i+1}$ is in $C_{0} \cup C_{r}$ (note that one of $y_{i}-y_{i+1}$ and $y_{i+1}-y_{i}$ is a power of $\alpha$ and hence in $C_{0}$, while the other is in $C_{r}$ ).

Conversely, suppose that $\operatorname{ord}_{t}(p)=e<d$. Then $\mathbb{F}_{p}(\alpha)=\mathbb{F}_{p^{e}}$ which is a strict subfield of $\mathbb{F}_{q}$. Clearly $C_{0} \cup C_{r} \subseteq \mathbb{F}_{p^{e}}$. Suppose that $y_{1}, y_{2}, \ldots$ is an ordering of the elements of $\mathbb{F}_{q}$ such that adjacent elements always have a difference that is an element of $C_{0} \cup C_{r}$. Without loss of generality we can take $y_{1}=0$. But then every element $y_{i}$ is in the subfield $\mathbb{F}_{p^{e}}$, which is a contradiction. Hence, the desired ordering cannot exist.

It is interesting to note that the proof above does not depend on the structure of the starter $S$. Either all $r$-quotient starters in $\mathbb{F}_{q}$ yield sequentially uniform one-factorizations or they all do not do so.

Example 4.2. Let $q=25$ so that $t=3$ and $r=4$. We have $\operatorname{ord}_{t}(p)=2=d$, so Theorem 4.1 asserts that any 4-quotient starter will yield a sequentially uniform onefactorization. In particular, if we take $\mathbb{F}_{25}=\mathbb{Z}_{5}[x] /\left(x^{2}+x+2\right)$ then $C_{0}$ contains a basis $\{1, \alpha\}$ for the field, where $\alpha=x^{8}=3 x+1$. The field elements can be cyclically ordered

$$
0,1,2,3,4,3 x, \ldots, 3 x+4, x, \ldots, x+4,4 x, \ldots, 4 x+4,2 x, \ldots, 2 x+4,0
$$

so that the difference of consecutive elements is either 1 or $\alpha$. 
Most applications of $r$-quotient starters use values of $r$ that are powers of two (see, for example, [6]). It is interesting to determine the conditions under which the hypotheses of Theorem 4.1 are satisfied in this case. This is done in Lemma 4.3.

Lemma 4.3. Suppose $q=p^{d}$ is an odd prime-power (with p prime and $d>1$ ) such that $q=2^{k} t+1$ and $t>1$ is odd. Then one of the two following conditions hold:

1. $\operatorname{ord}_{t}(p)=d$, or

2. $p=2^{j}-1$ for some integer $j$ (i.e., $p$ is a Mersenne prime) and $d=2$. (In this case, $\operatorname{ord}_{t}(p)=1$ is less than d.)

Proof: Suppose that $p^{e} \equiv 1(\bmod t)$ for some positive integer $e<d$ (note that $\left.e \mid d\right)$. Let $p^{e}=b t+1$ where $b$ is a positive integer. Then

$$
2^{k} t+1=q=\left(p^{e}\right)^{d / e}=(b t+1)^{d / e}=c b t+1
$$

for some integer $c$. Hence, $b \mid 2^{k}$, and therefore $b=2^{\ell}$ for some positive integer $\ell \leq k$. So we have that $p^{e}=2^{\ell} t+1$.

Let $\rho=p^{e}$ and $f=d / e$. Then we have that

$$
t=\frac{\rho^{f}-1}{2^{k}}=\frac{\rho-1}{2^{\ell}} .
$$

Removing common factors, we obtain

$$
\rho^{f-1}+\rho^{f-2}+\cdots+\rho+1=2^{k-\ell} .
$$

Suppose that $k=\ell$. Then the right side of (1) is equal to 1 , so $f=1$ and $d=e$. This contradicts the assumption that $d>e$. Therefore $k>\ell$ and the right side of $(1)$ is even.

Now, suppose that $f$ is odd. Then the left side of (1) is odd, and we have a contradiction. Therefore $f$ is even, and $\rho+1$ is a factor of the left side of (1). This implies that $2^{k-\ell} \equiv 0(\bmod \rho+1)$, and hence $\rho=2^{j}-1$ for some integer $j \geq 2$. Then, after dividing (1) by the factor $\rho+1$, we obtain the following equation:

$$
\rho^{f-2}+\rho^{f-4}+\cdots+\rho^{2}+1=2^{k-\ell-j} .
$$

Suppose that $j<k-\ell$. Then the right side of $(2)$ is even and $\rho^{2}+1$ is a factor of the left side of $(2)$, so $\rho^{2}=2^{i}-1$ for some integer $i \geq 2$. But $\rho=2^{j}-1$ where $j \geq 2$, so $\rho \equiv 3$ $(\bmod 4)$. Then $\rho^{2} \equiv 1(\bmod 4)$, which contradicts the fact that $\rho^{2}=2^{i}-1$ where $i \geq 2$. Therefore we have that $j=k-\ell$. This implies that $f=2$ and so $d=2 e$. So $\rho=2^{j}-1$ for some integer $j$ and $q=\rho^{2}$.

However, it is easy to prove that the Diophantine equation $2^{u}-y^{v}=1$ has no solution in positive integers with $u, v>1^{\dagger}$. See, for example, Cassels [3, Corollary 2]. Therefore,

†This result is a special case of Catalan's Conjecture, which states that the Diophantine equation $x^{u}-y^{v}=1$ has no solution in positive integers with $u, v>1$ except for $3^{2}-2^{3}=1$. Catalan's Conjecture was proven correct in 2002 by Mihăilescu (see Metsänkylä [11] for a recent exposition of the proof).

THE ELECTRONIC JOURNAL OF COMBINATORICS 12 (2005), \#R1 
we can conclude that $\rho$ is prime. Hence, $p=2^{j}-1$ is a Mersenne prime, $e=1$ and $d=2$. In this case, we have $q=p^{2}$. Then we have that

$$
q-1=(p-1)(p+1)=(p-1) 2^{j} \equiv 0 \quad(\bmod t) .
$$

But $t$ is odd, so $p \equiv 1(\bmod t)$. Therefore $\operatorname{ord}_{t}(p)=1$.

Example 4.4. Let $q=961=31^{2}$ so $p=31$ and $d=2$. Here $p=31=2^{5}-1$ is a Mersenne prime. We can write $q=2^{5} 15+1$, so $t=15$. We see that $\operatorname{ord}_{t}(p)=1<2$, as asserted by Lemma 4.3.

The following corollary is an immediate consequence of Theorem 4.1 and Lemma 4.3.

Corollary 4.5. Suppose $q=p^{d}$ is an odd prime-power (with $p$ prime and $d>1$ ) such that $q=2^{k} t+1$ and $t>1$ is odd. Let $S$ be any $2^{k-1}$-quotient starter in $\mathbb{F}_{q}$. Then the one-factorization generated by $S$ can be ordered to be sequentially uniform if and only if it is not the case that $p$ is a Mersenne prime and $d=2$.

\section{Product construction}

We now recall the usual product construction for one-factorizations, and apply it to determine another infinite class of sequentially uniform one-factorizations.

Suppose that $F$ is a one-factor on $X$ and $G$ is a one-factor on $Y$, where $|X|=2 n$ and $|Y|=2 m$. Define various one-factors of $X \times Y$ by

$$
\begin{aligned}
F^{*} & =\left\{\left\{\left(x_{i}, y\right),\left(x_{i}^{\prime}, y\right)\right\}:\left\{x_{i}, x_{i}^{\prime}\right\} \in F, y \in Y\right\}, \\
G^{*} & =\left\{\left\{\left(x, y_{j}\right),\left(x, y_{j}^{\prime}\right)\right\}: x \in X,\left\{y_{j}, y_{j}^{\prime}\right\} \in G\right\}, \\
F G & =\left\{\left\{\left(x_{i}, y_{j}\right),\left(x_{i}^{\prime}, y_{j}^{\prime}\right)\right\}:\left\{x_{i}, x_{i}^{\prime}\right\} \in F,\left\{y_{j}, y_{j}^{\prime}\right\} \in G\right\} .
\end{aligned}
$$

Given one-factorizations $\mathcal{F}=\left\{F_{0}, \ldots, F_{2 n-2}\right\}$ and $\mathcal{G}=\left\{G_{0}, \ldots, G_{2 m-2}\right\}$ of $K_{2 n}$ and $K_{2 m}$ on the points $X$ and $Y$, respectively, it is easy to see that

$$
\begin{aligned}
\mathcal{F G}= & \left\{F_{i} G_{j}: i=0, \ldots, 2 n-2 \text { and } j=0, \ldots, 2 m-2\right\} \\
& \bigcup\left\{F_{i}^{*}: i=0, \ldots, 2 n-2\right\} \bigcup\left\{G_{j}^{*}: j=0, \ldots, 2 m-2\right\}
\end{aligned}
$$

is a one-factorization of $X \times Y$.

The following are easy lemmas about the cycle types of pairs of one-factors in $\mathcal{F} \mathcal{G}$.

Lemma 5.1. For any $i \in\{0, \ldots, 2 n-2\}$ and $j \in\{0, \ldots, 2 m-2\}$, the following all have cycle type $(4,4, \ldots, 4)$ :

(i) $F_{i}^{*} \cup G_{j}^{*}$

(ii) $F_{i} G_{j} \cup F_{i}^{*}$, and

(iii) $F_{i} G_{j} \cup G_{j}^{*}$. 
Lemma 5.2. If $\left(F_{0}, F_{1}, \ldots, F_{2 n-2}\right)$ is sequentially uniform of type $(4,4, \ldots, 4)$, then the following all have cycle type $(4,4, \ldots, 4)$ :

(i) $F_{i}^{*} \cup F_{i+1}^{*}$, and

(ii) $F_{i} G_{j} \cup F_{i+1} G_{j}$,

for any $i, j$, where the subscripts $i+1$ are reduced modulo $2 n-1$.

We can use the above results to give a product construction for sequentially uniform one-factorizations of type $(4,4, \ldots, 4)$.

Theorem 5.3. Suppose there exists a sequentially uniform one-factorization of $K_{2 n}$ of type $(4,4, \ldots, 4)$. Let $m \leq n$. Then there is a sequentially uniform one-factorization of $K_{4 m n}$ of type $(4,4, \ldots, 4)$.

Proof: We use all the notation above, with $\left(F_{0}, F_{1}, \ldots, F_{2 n-2}\right)$ sequentially uniform of type $(4,4, \ldots, 4)$ and $\mathcal{G}$ any one-factorization of $K_{2 m}$. The ordered one-factorization

$$
\begin{gathered}
\left(G_{0}^{*}, F_{0} G_{0}, F_{1} G_{0}, \ldots, F_{2 n-2} G_{0}, F_{2 n-2}^{*},\right. \\
G_{1}^{*}, F_{1} G_{1}, F_{2} G_{1}, \ldots, F_{0} G_{1}, F_{0}^{*}, \\
G_{2}^{*}, F_{2} G_{2}, F_{3} G_{2}, \ldots, F_{1} G_{2}, F_{1}^{*}, \\
\vdots \\
G_{2 m-2}^{*}, F_{2 m-2} G_{2 m-2}, \ldots, F_{2 m-3} G_{2 m-2}, F_{2 m-3}^{*}, \\
\left.F_{2 m-2}^{*}, F_{2 m-1}^{*}, \ldots, F_{2 n-3}^{*}\right)
\end{gathered}
$$

of $K_{4 m n}$ is sequentially uniform of type $(4,4, \ldots, 4)$ by Lemmas 5.1 and 5.2.

By applying the above product construction with $2 n$ a power of 2 - for which the existence of uniform one-factorizations of type $(4,4, \ldots, 4)$ are known - one immediately has the following corollary.

Corollary 5.4. For any odd integer $m \geq 1$, there is a sequentially uniform one-factorization of $K_{2^{t} m}$ of type $(4,4, \ldots, 4)$ for all integers $t \geq 2+\left\lceil\log _{2} m\right\rceil$.

Let $t_{0}=t_{0}(m)$ denote the smallest integer such that there is a sequentially uniform one-factorization of $K_{2^{t} m}$ of type $(4,4, \ldots, 4)$ for all integers $t \geq t_{0}$. Corollary 5.4 provides an explicit upper bound on $t_{0}(m)$; however, for a particular value of $m$, we might be able to give a better bound on $t_{0}(m)$. For example, the sequentially perfect one-factorization of $K_{4}$ shows that $t_{0}(1)=2$, the sequentially uniform one-factorization of $K_{12}$ of type $(4,4,4)$ given in Figure 1 yields $t_{0}(3)=2$, and the sequentially uniform one-factorization of $K_{20}$ of type $(4,4,4,4,4)$ exhibited in the Appendix gives $t_{0}(5)=2$. In fact, we conjecture that $t_{0}(m)=2$ for all odd integers $m \geq 1$.

As a final note, we observe that the existence results for sequentially uniform onefactorizations of $K_{2^{t} m}$ of type $(4,4, \ldots, 4)$ provide an interesting contrast to those for uniform one-factorizations of $K_{2^{t} m}$ of type $(4,4, \ldots, 4)$, which exist only when $m=1$ (see Cameron [2, Proposition 4.3]). 


\section{Acknowledgements}

The authors would like to to thank Dan Archdeacon, Cameron Stewart and Hugh Williams for useful discussions and pointers to the literature.

D. Stinson's research is supported by the Natural Sciences and Engineering Research Council of Canada through the grant NSERC-RGPIN \#203114-02. P. Dukes was supported by an NSERC post-doctoral fellowship.

\section{References}

[1] L.D. Andersen. Factorizations of graphs. In The CRC Handbook of Combinatorial Designs, C.J. Colbourn and J.H. Dinitz, eds., CRC Press, Boca Raton, 1996, pp. 653-666.

[2] P.J. Cameron. Minimal edge-colourings of complete graphs. J. London Math. Soc. 11 (1975) 337-346.

[3] J.W.S. Cassels. On the equation $a^{x}-b^{y}=1$. Amer. J. Math. 75 (1953), 159-162.

[4] J.H. Dinitz and P. Dukes. Some new perfect one-factorizations of the complete graph. University of Vermont Mathematics Research Report No. 2004-01.

[5] J.H. Dinitz, D.K. Garnick, and B.D. McKay. There are 526, 915, 620 nonisomorphic one-factorizations of $K_{12}$. J. Combin. Des. 2 (1994), 273-285.

[6] J.H. Dinitz and D.R. Stinson. Some new perfect one-factorizations from starters in finite fields. J. Graph Theory 13 (1989), 405-415.

[7] N.J. Finizio. Tournament designs balanced with respect to several bias categories. Bull. Inst. Combin. Appl. 9 (1993), 69-95.

[8] M.J. Grannell, T.S. Griggs, and J.P. Murphy. Some new perfect Steiner triple systems. J. Combin. Des. 7 (1999), 327-330.

[9] V. Leck. Personal communication, 2001. (See http://www.emba.uvm.edu/ 〜initz/newresults.html.)

[10] E. Mendelsohn and A. Rosa. One-factorizations of the complete graph - A survey. J. Graph Theory 9 (1985), 43-65.

[11] T. Metsänkylä. Catalan's Conjecture: Another old Diophantine problem solved. Bull. Amer. Math. Soc. 41 (2004), 43-57.

[12] A.P. Petrenyuk and A.Ya. Petrenyuk. Intersection of perfect 1-factorizations of complete graphs (Russian). Cybernetics 1980 6-8, 149. 
[13] E. Seah. Perfect one-factorizations of the complete graph - a survey. Bull. Inst. Combin. Appl. 1 (1991), 59-70.

[14] W.D. Wallis. One-factorizations of the complete graph. In Contemporary Design Theory: A Collection of Surveys, J.H. Dinitz and D.R. Stinson, eds., John Wiley \& Sons, New York, 1992, pp. 593-631.

[15] W.D. Wallis. One-factorizations. Kluwer Academic Publishers, Dordrecht, NL, 1997.

[16] I.M. Wanless. Atomic Latin squares based on cyclotomic orthomorphisms. Submitted.

[17] J.Z. Zhang. Some results on perfect 1-factorizations of $K_{2 n}$. Dianzi Keji Daxue Xuebao 21 (1992), 434-436. 


\section{Appendix}

Below is a table giving all possible types for sequentially uniform one-factorizations of $K_{2 n}$, $14 \leq 2 n \leq 24$. Each type is realized by the ordered 1-factorization $F_{S}(1)$ corresponding to the starter $S=\left\{\left\{x_{i}, x_{i}+i\right\}: i=1, \ldots, n-1\right\}$ in $\mathbb{Z}_{2 n-1}$.

\begin{tabular}{|c|c|}
\hline type & $\left(x_{1}, \ldots, x_{n-1}\right)$ \\
\hline \hline$(14)$ & $(1,5,8,6,12,3)$ \\
\hline$(10,4)$ & $(1,9,4,6,3,12)$ \\
\hline$(8,6)$ & $(1,5,9,6,3,11)$ \\
\hline$(6,4,4)$ & $(7,2,9,1,6,10)$ \\
\hline \hline$(16)$ & $(1,4,8,9,7,14,3)$ \\
\hline$(12,4)$ & $(1,4,8,9,5,12,7)$ \\
\hline$(10,6)$ & $(1,7,11,4,5,12,6)$ \\
\hline$(8,8)$ & $(1,3,7,9,6,8,12)$ \\
\hline$(8,4,4)$ & $(2,11,9,4,5,1,14)$ \\
\hline$(6,6,4)$ & $(3,11,2,6,7,8,9)$ \\
\hline$(4,4,4,4)$ & $(3,6,11,12,5,7,2)$ \\
\hline \hline$(18)$ & $(1,3,7,12,8,9,4,6)$ \\
\hline$(14,4)$ & $(1,3,11,8,4,10,6,7)$ \\
\hline$(12,6)$ & $(1,5,8,12,9,4,13,15)$ \\
\hline$(10,8)$ & $(1,3,6,11,8,10,7,4)$ \\
\hline$(10,4,4)$ & $(1,13,5,7,9,4,16,12)$ \\
\hline$(8,6,4)$ & $(1,4,12,5,8,10,7,3)$ \\
\hline$(6,6,6)$ & $(1,6,10,5,11,15,7,12)$ \\
\hline$(6,4,4,4)$ & $(3,7,12,2,8,10,11,14)$ \\
\hline \hline$(20)$ & $(1,3,6,11,13,8,10,4,7)$ \\
\hline$(16,4)$ & $(1,3,9,13,10,8,4,18,16)$ \\
\hline$(14,6)$ & $(1,3,9,7,13,8,10,15,16)$ \\
\hline$(12,8)$ & $(1,3,9,13,6,10,8,18,14)$ \\
\hline$(12,4,4)$ & $(1,5,12,6,9,17,11,8,13)$ \\
\hline$(10,10)$ & $(1,3,12,9,11,4,7,17,18)$ \\
\hline$(10,6,4)$ & $(1,4,10,11,7,18,9,14,8)$ \\
\hline$(8,8,4)$ & $(1,3,12,10,6,7,16,9,18)$ \\
\hline$(8,6,6)$ & $(1,4,5,13,9,10,11,7,3)$ \\
\hline$(8,4,4,4)$ & $(2,5,11,4,10,12,13,9,16)$ \\
\hline$(6,6,4,4)$ & $(2,14,8,13,7,4,18,1,15)$ \\
\hline$(4,4,4,4,4)$ & $(6,16,17,11,4,8,3,5,12)$ \\
\hline & \\
\hline$, 4,4)$ & \\
\hline &
\end{tabular}

\begin{tabular}{|c|c|}
\hline type & $\left(x_{1}, \ldots, x_{n-1}\right)$ \\
\hline \hline$(22)$ & $(1,3,4,10,11,13,8,12,9,17)$ \\
\hline$(18,4)$ & $(1,3,4,13,11,12,8,6,10,20)$ \\
\hline$(16,6)$ & $(1,3,4,10,11,12,13,9,6,19)$ \\
\hline$(14,8)$ & $(1,3,4,12,9,11,13,10,6,19)$ \\
\hline$(14,4,4)$ & $(1,4,7,11,12,14,19,8,9,3)$ \\
\hline$(12,10)$ & $(1,3,6,11,13,10,12,20,8,4)$ \\
\hline$(12,6,4)$ & $(1,3,7,15,11,8,13,4,9,17)$ \\
\hline$(10,8,4)$ & $(1,3,7,11,14,6,13,9,16,8)$ \\
\hline$(10,6,6)$ & $(1,3,7,16,12,8,6,11,9,15)$ \\
\hline$(10,4,4,4)$ & $(1,5,11,13,19,6,8,10,16,20)$ \\
\hline$(8,8,6)$ & $(1,3,12,6,13,14,4,9,7,19)$ \\
\hline$(8,6,4,4)$ & $(1,3,10,16,12,9,4,6,19,8)$ \\
\hline$(6,6,6,4)$ & $(1,8,6,11,12,19,13,18,7,14)$ \\
\hline$(6,4,4,4,4)$ & $(1,11,5,16,9,6,17,10,19,15)$ \\
\hline \hline$(24)$ & $(1,3,4,9,11,15,12,14,8,10,18)$ \\
\hline$(20,4)$ & $(1,3,4,13,10,16,12,6,11,8,21)$ \\
\hline$(18,6)$ & $(1,3,4,10,16,13,8,9,11,12,18)$ \\
\hline$(16,8)$ & $(1,3,4,10,12,15,11,8,13,19,9)$ \\
\hline$(16,4,4)$ & $(1,3,6,10,16,11,15,12,4,8,19)$ \\
\hline$(14,10)$ & $(1,3,4,13,16,8,11,12,6,9,22)$ \\
\hline$(14,6,4)$ & $(1,3,6,10,12,16,13,11,21,8,4)$ \\
\hline$(12,12)$ & $(1,3,4,10,12,16,13,11,6,8,21)$ \\
\hline$(12,8,4)$ & $(1,3,4,13,10,12,9,14,20,11,8)$ \\
\hline$(12,6,6)$ & $(1,3,4,15,9,10,11,12,13,21,6)$ \\
\hline$(12,4,4,4)$ & $(1,3,13,15,4,6,14,10,8,20,11)$ \\
\hline$(10,10,4)$ & $(1,3,6,15,16,8,11,12,4,7,22)$ \\
\hline$(10,8,6)$ & $(1,3,4,11,9,16,12,13,8,10,18)$ \\
\hline$(10,6,4,4)$ & $(1,3,4,12,15,13,10,6,9,21,11)$ \\
\hline$(8,8,8)$ & $(1,3,4,11,16,8,10,12,13,9,18)$ \\
\hline$(8,8,4,4)$ & $(1,3,19,14,10,7,4,12,8,6,21)$ \\
\hline$(8,6,6,4)$ & $(1,3,9,13,22,15,7,10,11,6,8)$ \\
\hline$(8,4,4,4,4)$ & $(1,4,16,10,13,9,5,22,17,11,20)$ \\
\hline$(6,6,6,6)$ & $(1,5,6,12,15,21,10,11,13,8,3)$ \\
\hline$(6,6,4,4,4)$ & $(1,6,14,7,13,15,3,12,19,22,16)$ \\
\hline$(4,4,4,4,4,4)$ & $(5,17,9,11,13,21,1,22,16,10,3)$ \\
\hline & \\
\hline &
\end{tabular}

